\title{
Compound conditioning: Component strength in a compound CS as a function of test trial ratio
}

\author{
DAVID C. BLOUIN and A. GRANT YOUNG \\ Louisiana State University, Baton Rouge, Louisiana 70803
}

\begin{abstract}
An active avoidance conditioning paradigm was used in training 48 male albino rats to a compound CS, light and tone. Four groups of subjects, each divided into three subgroups, were overtrained to the compound with intervening test trials to the compound, tone, or light according to the ratios $1 / 60,1 / 30$, $1 / 10,1 / 5$. The same CS was given on all test trials. After overtraining, each subject received an extinction session to all stimuli. The results showed the component response strengths increased during overtraining. On the first test trial, the response strength to the compound was greatest, whereas on the last test trial, the response strengths to the compound, light, and tone were similar. The effects of test trial ratios were minimal.
\end{abstract}

Many investigators have been concerned with the response strength of components in a compound $\mathrm{CS}$ as a function of the number of training trials to the compound (Razran, 1965; Toporkova, 1961; Wickens, Wickens, \& Nield, Note 1). These studies employed within-subjects designs that involved nonreinforced test trials to the components during successive stages of active avoidance training. The findings were consistent, showing that the response strength of components decreased as a function of training. The results have been interpreted in terms of stimulus configuration, that is, a synthesis of the components into a single functional CS due to extinction of the components via nonreinforced test trials (Razran, 1965).

Studies that employed between-subjects designs have produced conflicting results in showing that the response strength of components increased as a function of avoidance training (Baker, 1968, 1969). In between-subjects designs, subjects are given varying amounts of training prior to nonreinforced test trials to the components. Baker (1969) also used a within-subjects comparison group where the test trial ratio was less than the ratios used in past studies. The within-subjects and betweensubjects comparisons were not directly comparable. The evidence did not support the stimulus configuration hypothesis in either the within- or between-subjects comparison groups. However, as all studies have shown, the response strength of components was always less than the response strength of the compound. The differential response strength was expected merely on the basis of stimulus generalization, that is, a change in stimulus conditions from training trial to test trials.

The present study incorporated within- and betweensubjects designs, such that the within- and betweensubjects comparison groups were comparable. The major

This research was supported in part by a grant from the Graduate Council On Research, LSU, to the second author. concern was to test the stimulus configuration hypothesis as a function of test trial ratio during the overtraining period in an active avoidance paradigm. The expectation was that larger ratios would provide supportive evidence for stimulus configuration (Razran, 1965; Toporkova, 1961; Wickens, Wickens, \& Nield, Note 1). Second, the response strength of components was expected to be greater for subjects that were given more training trials to the compound, but the same frequency of test trials (Baker, 1969). A third hypothesis was that presentation of a single component would elicit an orienting reflex (OR) which would inhibit the response to the components (Baker, 1968; Thompson \& Shaw, 1965). The test trials would initially serve to extinguish the OR, allowing the response strength of the components to be demonstrated. Additional test trials would serve to decrease the response strength of the components.

\section{METHOD}

Subjects

The subjects were 48 naive male albino rats, approximately 200-225 $\mathrm{g}$ in weight at the start of the experiment.

\section{Apparatus}

The apparatus consisted of a one-way active avoidance chamber (19 x 5 x 6.5 in.), constructed of $1 / 4$-in. plywood, with a white danger compartment and a black safety compartment. The floor of the danger compartment was a grid which could be electrified. All experimenter-controlled events were operated by an electronic programming device. The compound CS consisted of a simultaneous light and tone whose sources were located on opposite sides of the grid compartment. The light CS consisted of two 7-W light bulbs. The tone CS was a $1,000-\mathrm{Hz}$ tone with an intensity of $2 \mathrm{~dB}$ above a background measure of $60 \mathrm{~dB}$. The UCS was a $1.0-\mathrm{mA}$ shock produced by a shock generator. The UCS remained on until the subject escaped from the danger compartment. Response latencies and intertrial intervals were automatically timed with a stop clock. 


\section{Procedure}

The subjects were selected randomly from the LSU colony, housed in individual cages, and maintained on ad-lib food and water for a period of 5 days prior to the start of the experiment. Four days prior to being used in the experiment, the subjects were handled in groups of four for approximately $5 \mathrm{~min}$ daily. All subjects participated in one training session and one extinction session.

A one-way active avoidance conditioning procedure was used. All subjects were initially given a 5 -min adaptation period in the avoidance chamber before conditioning began. The compound CS was $3 \mathrm{sec}$ in duration, the components beginning and terminating simultaneously. The onset of the UCS immediately followed the compound CS termination. A response-terminated procedure was used with both the CS and UCS, that is, once the subject passed over the barrier, the CS or UCS terminated. A 30 -sec intertrial interval was used.

The subjects were randomly divided into four groups $(n=12)$, with each group divided randomly into three subgroups $(n=4)$. All subjects were initially given avoidance training to a criterion of three out of four consecutive avoidance responses before CS offset. Following criterion level, all subjects were given 60 overtraining trials with varying ratios of test trials to either the compound $\mathrm{CS}(\mathrm{C})$, the tone component $(\mathrm{T})$, or the light component (L). The subjects in each subgroup were always given test trials to the same CS.

The subjects in Group 1 received 59 overtraining trials to the compound, with Trial 60 being a test trial to either the compound (Subgroup G1C), the tone component (Subgroup G1T), or the light component (Subgroup G1L). The test trial ratio during overtraining for Group 1 was $1 / 60$. Group 2 received a test trial on Trials 30 and 60 of the overtraining period, the subgroups being G2C, G2T, and G2L, respectively. The test trial ratio for Group 2 was $1 / 30$. Group 3 received a test trial on every 10th trial of the overtraining period, the subgroups being G3C, G3T, and G3L, respectively. The test trial ratio for Group 3 was $1 / 10$. Group 4 received a test trial on every fifth trial of the overtraining period, the subgroups being G4C, G4T, and G4L, respectively. The test trial ratio for Group 4 was $1 / 5$.

On all training and test trials response latencies were taken. If on a test trial the subject did not respond within $2 \mathrm{sec}$ after CS offset, the latency was recorded as 5 sec. Twenty-four hours after overtraining, all subjects were subjected to an extinction session in which the stimulus presented on each trial was randomly selected. The extinction criterion was five consecutive no-response trials which were recorded as latencies of $5 \mathrm{sec}$.

\section{Design}

Several analyses of variance were conducted. A critical ratio difference (CRD), with the three subgroups of subjects nested within each of the four test trial ratio groups, was used to ascertain differences in the mean trials to criterion for the acquisition period. A separate CRD, with a 4 by 3 factorial arrangement of treatments, was used to ascertain differences in the mean response latencies on the first test trial of the overtraining period, in the mean response latencies on the last test trial of the overtraining period, and in the mean trials to extinction. The four-leveled factor was the test trial ratio and the three-leveled factor was the stimulus presented on test trials. A CRD that involved a split-plot arrangement of treatments was used to ascertain differences in the mean response latencies on common test trials. One analysis involved a 2 by 3 factorial arrangement of the ratios $(1 / 10$ and $1 / 5)$ and the three stimulus types as the main factors, with the common test trials $(10,20$, $30,40,50,60)$ as the levels of the subfactor. A second analysis involved a 3 by 3 factorial arrangement of the ratios $(1 / 30,1 / 10$, and $1 / 5)$ and the three stimulus types as the main factors, with the test trials (30 and 60 ) as the subfactors. A stepwise regression procedure was used to determine the best model for describing the change in response latencies as a function of test trial number. The independent factors were the linear, quadratic, and cubic components.

\section{RESULTS}

The results for the mean trials to criterion for the initial acquisition period showed that the subjects in the four test trial ratio groups did not differ significantly in the mean trials to criterion $(\mathrm{p}>.05)$. The mean trials to criterion for Group 1 (1/60), Group $2(1 / 30)$, Group $3(1 / 10)$, and Group $4(1 / 5)$ were 7.33, 6.25, 7.08 , and 6.33 , respectively. The three subgroups within each ratio group did not differ significantly in the mean trials to criterion.

The results for the mean response latencies on the first test trial of the overtraining period showed that the stimulus presented on the first test trial had a significant effect at the .01 level. The first test trial was Trial 60 for Group 1, Trial 30 for Group 2, Trial 10 for Group 3, and Trial 5 for Group 4. The mean response latencies for the compound CS, tone CS, and the light CS were .80, 1.05 , and $1.71 \mathrm{sec}$, respectively. A priori comparisons with Duncan's $t$ tests showed that the mean response latencies for each stimulus CS condition were significantly different from each other $(p<.05)$. The different amounts of overtraining prior to the first test trial did not have a significant effect on the mean response latencies on the first test trial.

The results for the mean response latencies on the last test trial of overtraining, that is, Trial 60 for all subjects, showed that there were no differences in the average latencies to the three stimulus CS conditions. The different numbers of test trials did not produce significant differences in the mean latencies on the last test trial. The stimulus conditions and test trial ratios did not result in significant differences in the mean trials to extinction.

The results for the mean response latencies, with the common test trials as the subfactor, revealed no significant trial effect. One regression analysis showed that the linear, quadratic, and cubic components for test trials provided the best model for describing the change in response latencies as a function of the test trial number. The behavior of subjects that received the tone CS on every 10th trial decreased in a cubic relationship.

\section{DISCUSSION}

The major concern of the present study was to test the stimulus configuration hypothesis. The results provided two lines of evidence against the stimulus configuration hypothesis. First, the two largest test trial ratios did not produce a progressive decline in the response strength of the components, as predicted from the hypothesis. In addition, the data from the extinction trials did not show evidence that supported the stimulus configuration hypothesis. If the function of test trials was to decrease the response strength of the components, then the higher ratios of test trials should have led to fewer trials to extinction. No 
differences were found in the effects of test trial ratios either in overtraining or extinction.

The type of stimulus CS that was presented on the first test trial showed a differential effect on response latencies. The response latencies to the components were longer than the latencies to the compound on the first test trial. The fact that a decrement occurred on the first, but not on the last, test trial is opposite to the prediction that was derived from the stimulus configuration hypothesis. A hypothesis based on stimulus generalization predicted the initial decrement as well as a decrement on the last test trial. The evidence led to the conclusion that neither a hypothesis based on stimulus configuration nor on stimulus generalization provided an adequate account of the results.

The findings of the present study were in partial agreement with the findings reported by Baker $(1968,1969)$. The OR model accounted for the response decrement that occurred to the components on the first test trial. The number of test trials needed to extinguish the OR was found to be minimal. The hypothesis that the function of overtraining was to increase the response strength of the components gained indirect support. The lack of a progressive increase in the response strength of the components indicated that the performance of subjects was near optimal subsequent to the acquisition period. In general, the lack of significant trial effects indicated that the level of performance was maintained across test trials to either the compound or the components. The inference is that once the OR was extinguished, the compound and components demonstrated functional equivalance.

The implication is that the generalization gradient significantly declined under the high motivational level of the subjects. A clear demonstration of an increase or decrease in component strength as a function of training may depend on the presentation of even higher test trial ratios relatively early in training.
The conclusion is that once the components were combined into a compound CS, the components were responded to in a similar manner.

\section{REFERENCE NOTE}

1. Wickens, D. D., Wickens, C. D., \& Nield, A. F. Conditioning, extinction, and spontaneous recovery of the GSR, breathing and leg withdrawal in the cat. Paper presented at the meeting of the Psychonomic Society, Chicago, October 1965.

\section{REFERENCES}

BAKER, T. W. Properties of compound conditioned stimuli and their components. Psychological Bulletin, 1968, 70, 611-625.

BAKER, T. W. Component strength in a compound CS as a function of number of acquisition trials. Journal of Experimental Psychology, 1969, 79, 347-352.

RAzRAN, G. Empirical codifications and specific theoretical implications of compound-stimulus conditioning: Perception. In W. F. Prokasy (Ed.), Classical conditioning. New York: Appleton-Century-Crofts, 1965.

Thompson, R. F., \& SHAw, J. A. Behavioral correlates of evoked activity recorded from association areas of the cerebral cortex. Journal of Comparative and Physiological Psychology, 1965, 60, 329-340.

Toporkova, L. A. Formation of conditioned respiratory reflexes to simultaneous and successive complex of stimuli in cats. Pavlov Journal of Higher Nervous Activity, 1961, 11, 103-106.

(Received for publication November 22, 1976.) 